\title{
Postoperative thromboembolic prophylaxis in joint replacement surgery: Guidelines and daily practice
}

\author{
Arina J ten Cate-Hoek ${ }^{*}$ and Karly Hamulyák
}

\begin{abstract}
This is a commentary discussing the article published in Thrombosis Journal by Subramanian et al. Thrombosis Journal 2012, 10:15].
\end{abstract}

The need for post-operative thromboembolic prophylaxis in orthopaedic surgery is beyond debate. Postoperative thrombosis is prevalent in these patients. In the absence of thromboprophylaxis, the incidence of venographically confirmed deep vein thrombosis (DVT) in the first 7 to 14 days following surgery is about $40-60 \%$. About half of these thrombi become symptomatic. In most cases DVT is diagnosed within a period of two months after the patients' discharge from hospital [1].

Guidelines on prophylaxis of venous thromboembolism that have been published by at least four different organisations established in the field of medical research, all agree on the need for post-operative prophylaxis [2-5].

Current prophylactic treatment is highly effective, and a significant reduction in the venous thromboembolism (VTE) incidence can therefore be reached by the use of adequate prophylaxis $[6,7]$.

However, despite the various guideline recommendations and readily available efficacious medication, the use of preventive measures against VTE in clinical practice has been found to be sub-optimal in several instances. A retrospective study by $\mathrm{Yu}$ et al. [8] found that the overall compliance with (ACCP) guidelines for the prevention of VTE in six American hospitals was only $13.3 \%$, ranging from $2.8 \%$ for neurosurgery to $52.4 \%$ for orthopaedic surgery. A survey amongst surgeons in the United Kingdom by Sharif et al. [9] showed

* Correspondence: arina.tencate@maastrichtuniversity.n

Department of Internal medicine, Maastricht University Medical Center and

Cardiovascular Research Institute (CARIM), Maastricht, the Netherlands that only $7 \%$ of respondents were adhering to NICE guidance after hip fracture surgery and $44 \%$ after hip arthroplasty.

Therefore, the stated rationale and considerations by the authors of the article published Thrombosis Journal on the 12 month review of a modified protocol using low dose Dabigatran etexilate in postoperative thromboembolic prophylaxis in joint replacement surgery, to deviate from the NICE guideline, seem legitimate [10]. On the one hand, the deviation from the guidelines is in favour of increased compliance with protocol by inhospital (junior) staff. On the other hand, enhanced patient compliance and adherence in the out of hospital setting is anticipated by avoiding the need for self administered injections at home.

Usually guidelines fall behind with innovations in clinical practice. After the introduction of new treatment modalities some time passes before synthesized evidence finds its way into guideline recommendations. In the case of the novel oral anticoagulant drugs such as dabigatran and rivaroxaban this implementation into guidelines has been relatively swift.

At this moment there is lack of day-to-day clinical experience with the newly introduced drugs and therefore clinical evidence is less unequivocal. Clinical practice usually differs from the setting of a clinical trial. Post surgical gastrointestinal upset and vomiting may reduce the effectiveness of oral antithrombotic therapy. To start prophylactic treatment post surgery with subcutaneous administered LMWH is therefore an attractive alternative. 
The choice of the authors for a combination treatment of two individually established safe and efficient therapeutic treatment modalities is understandable, but has a number of methodological shortcomings. Patient management was instituted without any preliminary studies, such as to identify the optimal, safe and effective, dosing strategy for the overlap of two different treatment modalities. In addition, a pragmatic, nonevidence based, choice was made for the single use of a low dose of $150 \mathrm{mg}$ dabigatran once daily, irrespective of patient characteristics such as bodyweight, age or kidney function. On the other hand, all consecutive patients who met the inclusion criteria were managed according to the protocol, minimising selection bias. Data on complications such as VTE, bleeding, transfusion rates, periprocedural infections and mortality were prospectively collected and reviewed by an independent reviewer to control for bias.

In parallel to the article by Subramanian et al., comparable clinical experience was gained in the United States, in this case in the setting of a clinical study, with similar alternative treatment strategies, bridging the periprocedural period with LMWH and making the transition to oral anticoagulant treatment before hospital discharge [11]. This study investigated the optimal interval for a safe overlap in anticoagulant activity between the LMWH and the oral anticoagulant drug rivaroxaban. The underlying rationale for the study being essentially the same: adapting the strategy to the requirements of day-to-day clinical practice.

The need for (observational) data on the novel oral anticoagulant drugs derived from clinical practice is widely recognized. Despite methodological shortcomings, the recent Subramanian et al. article adds to our knowledge on the everyday use of the novel oral anticoagulant medication and shows that the choice of prophylactic treatment does depend on clinical circumstances but also on patient preference.

Received: 30 August 2012 Accepted: 30 August 2012

Published: 3 September 2012

\section{References}

1. Falck-Ytter Y, Francis CW, Johanson NA, Curley C, Dahl OE, Schulman S, Ortel TL, Pauker SG, Colwell CW Jr: American College of Chest Physicians. Prevention of VTE in orthopedic surgery patients: Antithrombotic Therapy and Prevention of Thrombosis, 9th ed: American College of Chest Physicians Evidence-Based Clinical Practice Guidelines. Chest 2012, 141(2 Suppl):e278S-e325S.

2. Guyatt GH, Eikelboom JW, Gould MK, Garcia DA, Crowther M, Murad MH, Kahn SR, Falck-Ytter Y, Francis CW, Lansberg MG, AkI EA, Hirsh J: American College of Chest Physicians. Approach to outcome measurement in the prevention of thrombosis in surgical and medical patients: Antithrombotic Therapy and Prevention of Thrombosis, 9th ed: American College of Chest Physicians Evidence-Based Clinical Practice Guidelines. Chest 2012, 141(2 Suppl):e185S-e194S. Review.

3. Qaseem A, Chou R, Humphrey LL, Starkey M, Shekelle P: Clinical Guidelines Committee of the American College of Physicians Venous thromboembolism prophylaxis in hospitalized patients: a clinical practice guideline from the American College of Physicians. Ann Intern Med 2011, 155(9):625-632.

4. Scottish Inter-Collegiate Guideline Network (SIGN): Prevention and management of venous thromboeombolism. Edinburgh: SIGN; 2010. See http//www.sign.ac.uk/pdf/sign122.pdf

5. Treasure T, Hill J: NICE guidance on reducing the risk of venous thromboembolism in patients admitted to hospital. J R Soc Med 2010, 103(6):210-212.

6. Lereun C, Wells P, Diamantopoulos A, Rasul F, Lees M, Sengupta N: An indirect comparison, via enoxaparin, of rivaroxaban with dabigatran in the prevention of venous thromboembolism after hip or knee replacement. J Med Econ 2011, 14(2):238-244. Epub 2011 Mar 9. PubMed PMID: 21385145

7. Goff T, Kontakis G, Giannoudis PV: Safety and efficacy of rivaroxaban for thromboprophylaxis following lower limb surgery: an update. Expert Opin Drug Saf 2011, 10(5):687-696. Epub 2011 Mar 15. Review.

8. Yu HT, Dylan ML, Lin J, Dubois RW: Hospitals' compliance with prophylaxis guidelines for venous thromboembolism. Am J Health Syst Pharm 2007, 64(1):69-76.

9. Sharif KM, Rehman SF, Nunn T, Bagga TK: Implementation of the NICE guidelines for venous thromboprophylaxis; a national survey of hip surgeons. Hip Int 2009, 19(1):58-63.

10. Subramanian P, Kantharuban S, Shilston S, Pearce OJ: A 12 month review of a modified protocol using low dose dabigatran etexilate in postoperative thromboembolic prophylaxis in joint replacement surgery. Thromb J 2012, 10:14.

11. Mills RM, Berkowitz RD, Damaraju CV, Jennings LK, Wildgoose P: Initiation of rivaroxaban following low molecular weight heparin for thromboprophylaxis after total joint replacement: The Safe, Simple Transitions (SST) study. Thromb Res 2012. [Epub ahead of print] PubMed PMID: 22857800.

\section{doi:10.1186/1477-9560-10-18}

Cite this article as: ten Cate-Hoek and Hamulyák: Postoperative thromboembolic prophylaxis in joint replacement surgery: Guidelines and daily practice. Thrombosis Journal 2012 10:18.

\section{Submit your next manuscript to BioMed Central and take full advantage of:}

- Convenient online submission

- Thorough peer review

- No space constraints or color figure charges

- Immediate publication on acceptance

- Inclusion in PubMed, CAS, Scopus and Google Scholar

- Research which is freely available for redistribution 